\title{
Lidil
}

Revue de linguistique et de didactique des langues

47 | 2013

Le verbe pour exprimer le temps

\section{L'apprentissage des temps verbaux en espagnol. Relations entre connaissances déclaratives et usage dans les textes}

Carmen Rodríguez Gonzalo

\section{OpenEdition}

Journals

Édition électronique

URL : http://journals.openedition.org/lidil/3263

DOI : $10.4000 /$ lidil.3263

ISSN : 1960-6052

Éditeur

UGA Éditions/Université Grenoble Alpes

Édition imprimée

Date de publication : 31 mai 2013

Pagination : 41-59

ISBN : 978-2-84310-247-9

ISSN : $1146-6480$

Référence électronique

Carmen Rodríguez Gonzalo, «L'apprentissage des temps verbaux en espagnol. Relations entre connaissances déclaratives et usage dans les textes », Lidil [En ligne], 47 | 2013, mis en ligne le 01 décembre 2014, consulté le 01 mai 2019. URL : http://journals.openedition.org/lidil/3263 ; DOI : 10.4000/lidil.3263 


\title{
L'apprentissage des temps verbaux en espagnol. Relations entre connaissances déclaratives et usage dans les textes
}

\author{
Carmen Rodríguez Gonzalo*
}

\begin{abstract}
RÉSUMÉ
L'article présente une enquête sur la relation entre la connaissance déclarative des élèves sur les temps verbaux du passé en espagnol et son utilisation dans des activités d'écriture, avec la médiation d'une séquence d'enseignement de grammaire mise en œuvre dans le contexte naturel d'une classe de 4' d'ESO (15-16 ans). La comparaison entre les données du produit (les textes des élèves) et celles du processus (les interactions en classe) permet d'analyser la façon dont le transfert a lieu entre la connaissance conceptuelle et l'utilisation de la langue. Les résultats montrent l'importance de la révision des textes comme activité d'analyse qui développe la conscience métalinguistique des élèves et augmente le contrôle sur leurs propres productions.
\end{abstract}

\begin{abstract}
This paper investigates the correlation between declarative knowledge of Spanish verbal past tenses and usage in writing activities performed in the context of the classroom, in 15 and 16 year-old learners (Spanish Compulsory Education students). The investigation was led within the frame of a grammar teaching sequence in the natural context of the classroom. This paper shows how the contrast between production data (the learners' texts) and process data (classroom interactions) sheds light on the transfer between conceptual knowledge and linguistic use. Overall, this paper demonstrates the relevance of text reviewing as an analytic activity that develops the learners' metalinguistic awareness and increases their control over their own productions.
\end{abstract}

* GREAL, Dpt. de Didàctica de la Llengua i la Literatura, Universitat de València (Espagne). 
Cet article présente une enquête sur la réflexion grammaticale en classe, sur les relations entre le savoir grammatical et l'usage de la langue autour d'un problème spécifique, l'utilisation des temps verbaux du passé en espagnol, étudié à partir d'une intervention didactique avec des

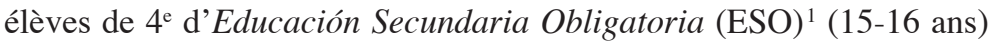
(Rodríguez Gonzalo, 2011) 2 .

Le point de départ a été un problème identifié en classe : la prédominance du passé simple (PS) dans les narrations écrites des élèves, dans des contextes où l'alternative avec le plus-que-parfait (PF) est possible (Perdió el libro que se compró/había comprado pocos días antes; «Il a perdu le livre qu'il a acheté/avait acheté quelques jours avant»). La préférence pour le PS au lieu du PF n'est pas une question strictement normative, mais plutôt une question entre usage spontané (surtout lié à l'oralité) et usage élaboré, ce qui survient le plus souvent dans la langue écrite et montre le degré de compétence des usagers. Nous proposons aux élèves une réflexion sur les divers usages des différentes formes verbales du passé, conçues comme un système organisé autour du PS (forme verbale absolue), comme dans l'exemple: FUI al cine el domingo, como HACÍA siempre; LLOVÍA y HABÍA QUEDADO con Luis y Alfredo en el parque, donde también nos REUNIRÍAmos con Marga y su prima, después de la película ${ }^{3}$.

\section{Cadre théorique et démarche méthodologique}

L'objectif général de la recherche est de contribuer à la compréhension des processus d'apprentissage de la grammaire et d'enquêter sur leur relation avec la compréhension et la production de textes, à partir d'un cas : l'enseignement et l'apprentissage des temps verbaux du passé dans l'enseignement obligatoire. Cet objectif se décompose en quatre

1. En Espagne, c'est la dernière classe du collège (enseignement obligatoire), mais en France, ce serait la première classe du baccalauréat, c'est-à-dire la classe de «seconde».

2. En espagnol, on peut consulter un autre travail sur ce thème (Rodríguez Gonzalo, 2011b), où nous présentons les bases théoriques et une version plus large des résultats de la thèse de doctorat.

3. En français, la forme verbale absolue serait le passé composé : «Je suis allé au cinéma le dimanche, comme je le faisais d'habitude; il pleuvait et j'avais un rendez-vous avec Luis et Alfredo dans le parc, où nous pourrions également rencontrer Marga et sa cousine, après le film.» 
sous-objectifs spécifiques : le premier, centré sur la perspective de l'apprentissage, est d'étudier comment la réflexion grammaticale se produit dans la classe, dans le contexte d'une séquence didactique de grammaire (SDG) conçue pour observer la relation entre la connaissance déclarative élaborée par les élèves sur les temps verbaux du passé et leur utilisation dans une activité d'écriture guidée didactiquement; le deuxième s'interroge sur la façon d'analyser l'activité en classe pour rendre compte de l'apprentissage et de l'activité métalinguistique des élèves; le troisième vise à installer les médiations didactiques qui favorisent la relation entre la réflexion et l'usage; enfin, le quatrième objectif propose la validation d'un modèle d'enseignement et d'apprentissage de la grammaire - les séquences didactiques de grammaire (SDGs), proposé en 2003 par Camps (Camps et coll., 2005; Camps \& Zayas, 2006) - qui favorise la réflexion métalinguistique dans ses diverses manifestations (Camps, 2000).

Le cadre théorique de notre enquête, qui s'inscrit dans une conception socio-constructiviste de l'enseignement-apprentissage, donne la priorité à la fois à l'activité réfléchie de l'élève en classe, entendue comme un espace d'interaction sociale, et à la réflexion du professeur sur le processus qu'on y développe. Du point de vue de l'apprentissage, nous avons considéré le savoir grammatical des élèves dans le cadre des savoirs conceptuels acquis à l'école et nous avons examiné les études sur les représentations des élèves et sur les obstacles, aussi bien en didactique de la langue (Kilcher-Hagedorn et coll., 1987; Camps et coll., 2001; Fisher, 2004) que dans d'autres didactiques. Dans la mesure où la connaissance grammaticale est un savoir conceptuel, nous avons tenu compte des études sur l'apprentissage de l'abstraction (Barth, 2004) et sur l'importance de l'interaction en classe pour parler des sciences (Lemke, 1997).

Par rapport aux contenus grammaticaux objet de l'enquête, ils ont fait l'objet d'une transposition didactique à partir d'une synthèse des études sur la temporalité en espagnol et sur l'organisation des temps en fonction de la perspective énonciative (Benveniste, 1959; Weinrich, 1974). Nous avons également considéré les questions relatives à la terminologie et à sa transposition didactique.

L'approche méthodologique de la recherche est qualitative. Nous avons combiné deux types de méthodes :

1. Le projet d'intervention a été conçu comme une étude d'évaluation à petite échelle (Piggot \& Barr, 2002), dont le but explicite est de valider un modèle pour améliorer l'instruction, dans ce cas, l'enseignement de la grammaire. Pour cela, nous développons une série d'activités 
guidées pour observer la manière dont les élèves apprennent dans un cadre scolaire dans une période limitée, en prenant le groupe-classe, et non des élèves individuels, comme unité d'analyse. Dans notre proposition, l'unité d'intervention est une SDG qui focalise l'attention sur le processus et non pas sur l'évaluation pré-et post-intervention.

2. La mise en œuvre de la séquence et l'analyse ultérieure des données est présentée comme une étude instrumentale de cas (Yin, 2003; Stake, 2005). Le cas est entendu comme l'instrument pour explorer la question en litige («issue», Stake, 2005, p. 26) qui, dans notre travail, se concentre sur les processus d'apprentissage de la grammaire et leur relation avec la production et la compréhension de textes.

Tout au long de l'enquête, la triangulation, comprise comme la combinaison de méthodes, des environnements et des perspectives théoriques (Flick, 2007, p. 243), nous permet de traiter sous plusieurs angles nos données et de renforcer nos hypothèses interprétatives. L'objectif, en définitive, vise à expliquer les relations complexes entre la théorie et la pratique au sein du triangle didactique.

Comme nous l'avons indiqué précédemment, l'intervention didactique est réalisée en proposant une SDG (figure 1 ) à 21 élèves de $4^{\mathrm{e}}$ ESO (15-16 ans), dans un collège public de la périphérie de Valence (Espagne), pendant douze séances de 55 minutes. Les séances sont enregistrées en audio et vidéo, en présence dans la classe de l'enseignant et du chercheur.

La SDG suit la démarche préconisée par Camps (2003). Le processus d'enseignement et d'apprentissage se déroule en trois phases : l'exploration du problème, l'enquête des élèves sur les temps verbaux du passé et la systématisation de l'apprentissage. Dans la deuxième phase, pour étudier si la réflexion sur les temps peut influencer l'amélioration de l'écriture en situation de classe, les élèves réalisent deux tâches communicatives (Nunan, 2002) conçues comme des petits projets de langue (Camps, 1996; Rodríguez Gonzalo, 2008) : l'une, de conceptualisation sur les formes verbales du passé (une affiche explicative) et l'autre, d'usage réfléchi, dans laquelle les élèves écrivent un petit récit.

L'analyse des données de ces tâches (indiquées à la figure 1) porte sur la façon dont a lieu le transfert des connaissances théoriques vers la pratique. Nous combinons ainsi le traitement quantitatif du corpus de textes d'élèves avec une analyse plus qualitative, de manière à identifier les acquis (épistémologiques et fonctionnels) et les obstacles que pose 
l'apprentissage grammatical des temps verbaux du passé, aussi bien dans la conceptualisation de ces temps que dans leur usage dans le récit.

\begin{tabular}{|c|c|c|}
\hline $\begin{array}{l}\text { Phase } 1 \\
\text { Quels temps verbaux du passé } \\
\text { utilise-t-on dans les récits? }\end{array}$ & $\begin{array}{l}\text { Phase } 2 \\
\text { Pourquoi utilise-t-on les temps } \\
\text { verbaux dans les récits ? }\end{array}$ & $\begin{array}{c}\text { Phase } 3 \\
\text { Un compte rendu sur l'usage des } \\
\text { temps verbaux du passé }\end{array}$ \\
\hline $\begin{array}{l}\text { Exposé des objectifs } \\
\text { d’apprentissage. } \\
\text { Act. 1. Identification et manipulation } \\
\text { des temps verbaux du passé. } \\
\text { Act. 2. Exploration de l'usage des } \\
\text { formes verbales en : } \\
\text { a. Genres oraux : narration, } \\
\quad \text { entretien radiophonique } \\
\text { (audition); } \\
\text { b. Genres écrits : récit } \\
\quad \text { littéraire (lecture) ; } \\
\text { Act. 3. Rédaction des conclusions } \\
\text { provisoires. }\end{array}$ & $\begin{array}{l}\text { Explication des contenus. } \\
\text { Act. 4. Élaboration d'une affiche } \\
\text { explicative sur les différences } \\
\text { entre deux temps verbaux à partir } \\
\text { de la lecture d'un dossier } \\
\text { théorique. Exposé oral en classe. } \\
\text { Act. 5. Rédaction d'un petit récit } \\
\text { sur une expérience vécue : } \\
\text { planification et première } \\
\text { rédaction. } \\
\text { Act. } 6 \text {. Exercice de choix du temps } \\
\text { verbal approprié aux contextes de } \\
\text { phrase établis. } \\
\text { Act. 7. Révision grammaticale } \\
\text { entre pairs de Pusage des temps } \\
\text { verbaux du passé dans les récits. } \\
\text { Révision de P'usage des temps } \\
\text { verbaux dans son propre récit. } \\
\text { Deuxième version. }\end{array}$ & $\begin{array}{l}\text { Act. 8. Planification d’un compte } \\
\text { rendu final écrit sur l'usage des } \\
\text { temps verbaux du passé. } \\
\text { Act. 9. Rédaction du compte rendu. } \\
\text { Act. } 10 \text {. Analyse du travail réalisé en } \\
\text { classe : apprentissages, difficultés } \\
\text { ou doutes, aspects intéressants. }\end{array}$ \\
\hline
\end{tabular}

Figure 1. - SDG : Pourquoi utilisons-nous les temps verbaux du passé?

\section{La conceptualisation des temps verbaux du passé}

\subsection{La tâche}

$\mathrm{Au}$ cours de la tâche, les lycéens, par paires, élaborent une affiche - comme soutien à une présentation orale - expliquant les résultats de leur réflexion autour de deux temps verbaux, tâche assignée par l'enseignant. La conception de l'affiche suit l'approche de Barth (2004) pour l'apprentissage des concepts. Selon l'auteur, la structure opérationnelle d'un concept se compose de la dénomination, des attributs et des exemples. La conceptualisation est considérée comme un processus cognitif qui peut être décomposé en plusieurs étapes : la perception (la discrimination sélective), la comparaison (processus analytique qui consiste à séparer une unité et ses composantes et à reconnaitre les relations entre elles), l'inférence et la vérification (la découverte d'une conclusion nouvelle à partir d'une source limitée de données). Ces aspects sont illustrés dans notre affiche ci-dessous (figure 2) : la première partie (caractérisation) comprend la conceptualisation de chaque forme verbale (la dénomination, les attributs sélectionnés et les exemples d'usage); la deuxième propose la comparaison des deux formes verbales. 


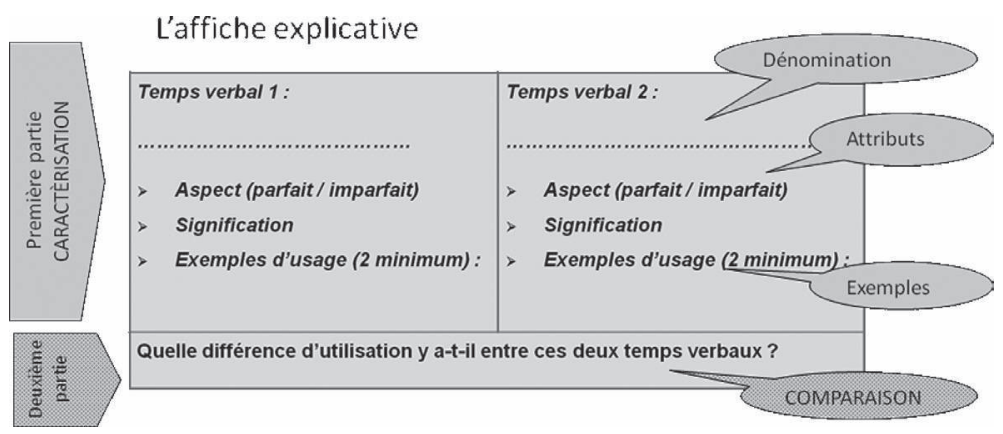

Figure 2. - L'affiche explicative et l'acquisition de concepts.

Nous avons analysé toutes les affiches réalisées par les élèves. Par rapport à la caractérisation des formes verbales du passé, les données prises en compte ont été les attributs ou traits caractéristiques (passé, action accomplie-non accomplie, antériorité-simultanéité-postériorité, forme verbale relative, d'autres valeurs comme la courtoisie...) et les exemples de chacune des formes verbales analysées.

Les résultats ont montré deux blocs bien différenciés : le passé simple (PS) et l'imparfait (PI) d'un côté et le plus-que-parfait (PF), le passé antérieur (PA) et le conditionnel présent (CP) de l'autre. Il semble que les élèves perçoivent le PS et le PI comme des formes absolues et les autres, comme des formes relatives. Bien que le PI puisse fonctionner comme une forme relative par rapport au passé simple, cette caractéristique n'est notée dans aucune des affiches.

Concernant les exemples, nous avons différencié les exemples valables des exemples non valables. Il existe des exemples valables de trois types: des exemples identiques à ceux proposés dans le dossier grammatical de la SDG; des exemples similaires, soit en imitant la structure syntaxique (Cuando fui a casa, tú ya habías llegado / "Quand je suis allé chez toi, tu étais déjà arrivé»), soit en utilisant la même marque temporelle (Ayer fui a comprar un bolígrafo / « Hier je suis allé acheter un stylo») et des exemples différents. Les exemples non valables sont des usages fautifs, c'est-à-dire, des usages agrammaticaux (*Hubisteis salido de casa cuando llegó el día / «*Vous fûtes sortis de chez vous quand le jour s'était levé») ou inadéquats des formes verbales du passé (Yo veía la televisión / «Je regardais la télée ${ }^{4}$ ).

4. L'usage de l'imparfait dans ce cas aurait besoin de complémentation temporelle : «Je regardais la télé quand... » ou «Je regardais la télé tous les jours». 
Concernant la comparaison entre les formes verbales du passé, nous avons tenu compte des traits distinctifs (selon les paires de formes verbales contrastées à chaque affiche) et nous avons analysé les stratégies utilisées pour la comparaison.

Les données de textes écrits ont été triangulées avec les interactions des élèves au cours de la tâche. Pour cela, sont prises en considération les interactions entre les élèves au cours des deux séances de travail consacrées à l'activité, formant ce que Lemke (1997) appelle le dialogue scientifique, défini sur la base de deux modèles : le modèle thématique (pour le contenu de l'interaction) et la structure de l'interaction sociale selon les participants (élève-élève, enseignant-élève...). Le modèle thématique permet d'observer comment les élèves parlent au cours de la conceptualisation et dans quelle mesure ils utilisent soit la langue spontanée, soit le métalangage grammatical.

\subsection{Les conclusions de la tâche de conceptualisation}

Nous indiquerons, principalement, nos conclusions sur les acquis et les obstacles relatifs à la conceptualisation.

En termes d'acquis, les traits qui permettent aux élèves de conceptualiser les différences des formes verbales du passé sont essentiellement l'aspect verbal (l'action qui se pose comme accomplie-non accomplie) pour le contraste entre le PS et le PI et l'antériorité-postériorité par rapport au PS comme forme absolue, pour le PF, le PA ou le CP. L'aspect (accompli-non accompli) est un trait que les élèves perçoivent plus facilement que l'antériorité-postériorité, qui implique en même temps la compréhension de la différence entre la forme absolue et les formes relatives dans l'organisation des temps du passé. Les formes verbales les mieux caractérisées par les informateurs sont le PS et le PF, pour la compréhension des traits grammaticaux et pour la plus grande maitrise de l'usage.

Dans la conceptualisation, l'application de traits aux exemples et la comparaison entre les différentes formes verbales semble essentielle. Avec ces deux opérations, on brise la dynamique du «dire la connaissance», en s'appropriant le discours des ouvrages de référence, et on oblige les apprenants à tester leur compréhension du métalangage grammatical. Il convient de noter que l'imitation des exemples se révèle une stratégie utile pour induire chez les élèves l'utilisation des structures syntaxiques et des marqueurs temporels qui mettent en évidence les caractéristiques énumérées pour chaque verbe. 
Par rapport aux obstacles identifiés, nous pouvons les regrouper en deux catégories : ceux qui correspondent à des difficultés de conceptualisation (obstacles épistémologiques) et ceux qui montrent le manque de maitrise de l'usage par les élèves. En ce qui concerne les obstacles épistémologiques, observés principalement dans trois temps verbaux : le PI, le PA et le CP, le trait le plus difficile à comprendre a été celui d'antériorité-simultanéité-postériorité (trait lié à la nature relative des formes verbales qui l'illustrent), pour sa difficulté théorique et aussi pour la nécessité de l'observer dans des structures syntaxiques complexes.

Les obstacles liés au manque de maitrise sont principalement concentrés dans le PA et le CP. Le PA est une forme non utilisée par les élèves; ils n'ont donc pas la maitrise suffisante pour en détecter les usages agrammaticaux. D'autre part, les valeurs modales du $\mathrm{CP}^{5}$ sont plus facilement perçues par les élèves et entravent la perception de cette forme verbale comme étant relative par rapport au PS ${ }^{6}$. Dans le PI, son usage comme forme verbale pour des actions habituelles entrave la perception de son trait de forme verbale simultanée du PS et même de forme verbale absolue par rapport à d'autres formes verbales du passé.

Ces données permettent une gradation dans la complexité de la caractérisation des formes verbales du passé, que nous reproduisons dans la figure 3 :

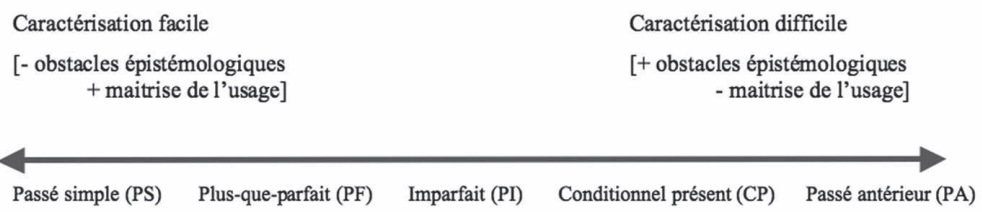

Figure 3. - Caractérisation des formes verbales du passé par les élèves.

\section{L'usage réfléchi des formes verbales du passé}

\subsection{La tâche}

L'activité d'élaboration d'un petit récit sur une expérience vécue vise à la réalisation d'une production planifiée dans laquelle les élèves utilisent consciemment les différentes formes verbales du passé, à la suite

5. Exemple : Sería muy inteligente, pero suspendió el examen. («Il serait très intelligent mais il a échoué à l'examen.»)

6. Exemple : Ella prometió que nos ayudaría. («Elle a promis qu'elle nous aiderait.») 
du travail antérieur de réflexion métalinguistique explicite (act. 4, fig. 1, expliquer les différences de sens et d'usage de deux temps verbaux du passé).

Comme stratégie didactique, nous avons proposé :

- la planification du texte, menée à terme en classe avec un modèle (fig. 4); ensuite, la rédaction individuelle de la première version du petit récit;

- la lecture à voix haute de ces versions au groupe-classe et une première réflexion sur les temps verbaux utilisés;

- un bref exercice sur les corrélations des temps du passé dans des contextes de phrases souvent utilisées et la révision entre pairs de l'usage des temps verbaux du passé;

- l'écriture individuelle de la seconde version du récit.

Le modèle de l'activité de planification est destiné à influencer indirectement l'emploi des temps, en marquant la différence entre les différents moments du passé (antérieurs et postérieurs au fait central de la narration) et entre les actions habituelles et ponctuelles.

Les temps verbaux du passé sont, dans ce cas-là, l'instrument que les élèves doivent utiliser pour écrire leur texte, mais la réflexion grammaticale n'est pas directement le sujet de l'écriture. Nous voulions voir si le passage d'une situation de «langage transparent» (raconter une expérience vécue) à une situation de «langage opaque» (observer et analyser les temps verbaux utilisés par un partenaire), à travers des tâches pédagogiques guidées au sein de la SDG, permettait aux élèves de surmonter la barrière entre le savoir pratique et le savoir réfléchi visé dans Camps et coll. (2005, p. 11) et de se positionner comme des usagers conscients des temps verbaux du passé.

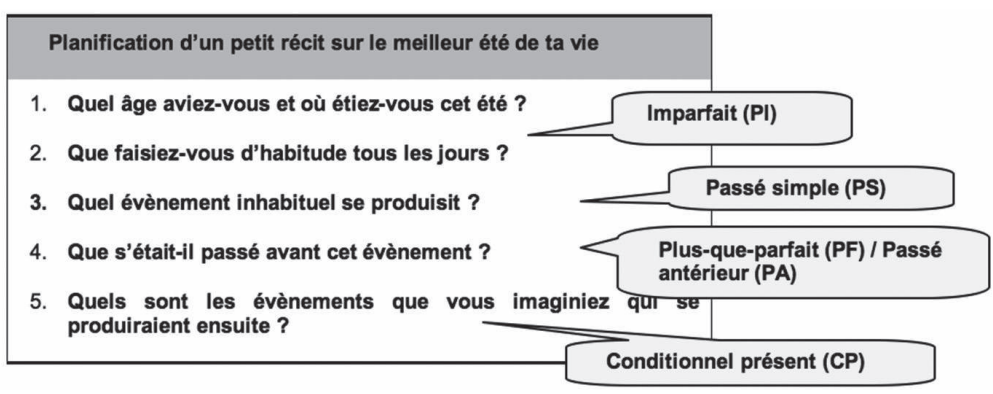

Figure 4. - Les temps verbaux implicites dans le modèle de planification du petit récit. 
Pour obtenir une interprétation plus précise de ce qui s'est passé dans la classe, nous avons combiné l'analyse quantitative de l'usage des temps verbaux du passé dans tous les textes des élèves, à chaque phase d'élaboration du récit, avec l'analyse qualitative des interactions de classe s'étant déroulées pendant la tâche.

Les données de la figure 5 montrent les temps verbaux utilisés dans les textes des élèves dans les trois phases du développement du récit : a) dans le modèle de planification; b) dans la première version du récit; c) à la deuxième version, après la révision des textes entre pairs.

Dans chaque cas, nous avons fait attention aux formes basiques de l'usage spontané (PS et PI) ou aux formes qui marquent l'action antérieure (PF ou PA) ou postérieure (CP) au PS.

\begin{tabular}{|c|c|c|c|c|c|c|c|}
\hline $\begin{array}{l}\text { Usage des temps } \\
\text { verbaux du passé }\end{array}$ & $\begin{array}{l}4 \text { temps } \\
\text { verbaux }\end{array}$ & $\begin{array}{l}3 \text { temps } \\
\text { verbaux }\end{array}$ & $\begin{array}{l}\text { Subtotal } \\
\text { style } \\
\text { soutenu }\end{array}$ & $\begin{array}{l}2 \text { temps } \\
\text { verbaux }\end{array}$ & $\begin{array}{l}1 \text { temps } \\
\text { verbal }\end{array}$ & D'autres cas & $\begin{array}{l}\text { Subtotal } \\
\text { usage } \\
\text { spontané }\end{array}$ \\
\hline $\begin{array}{l}\text { Planification } \\
\text { (21 élèves) }\end{array}$ & $\begin{array}{l}3 \text { cas } \\
(14,28 \%)\end{array}$ & $\begin{array}{l}4 \text { cas } \\
(19,04 \%)\end{array}$ & $\begin{array}{l}7 \text { cas } \\
(33,32 \%)\end{array}$ & $\begin{array}{l}10 \text { cas } \\
(47,61 \%)\end{array}$ & $\begin{array}{l}2 \text { cas } \\
(9,52 \%)\end{array}$ & $\begin{array}{l}2 \text { cas }(9,52 \%) \text { pas } \\
\text { de planification }\end{array}$ & $\begin{array}{l}14 \text { cas } \\
(66,65 \%)\end{array}$ \\
\hline $\begin{array}{l}\text { Première version } \\
\text { du récit } \\
\text { (21 élèves) }\end{array}$ & $\begin{array}{l}4 \text { cas } \\
(19,04 \%) \\
1 \text { cas } \\
\text { échoué }\end{array}$ & $\begin{array}{l}4 \text { cas } \\
(19,04 \%) \\
1 \text { cas } \\
\text { échoué }\end{array}$ & $\begin{array}{l}8 \text { cas } \\
(38,08 \%)\end{array}$ & $\begin{array}{l}4 \text { cas } \\
(19,04 \%)\end{array}$ & 0 & $\begin{array}{l}7 \text { cas }(33,33 \%) \\
\text { pas de } 1 \text { re version } \\
\text { du récit écrit + } 2 \\
\text { absences }(9,52 \%)\end{array}$ & $\begin{array}{l}13 \text { cas } \\
(61,89 \%)\end{array}$ \\
\hline \multicolumn{8}{|c|}{ RÉVISION ENTRE PAIRS } \\
\hline $\begin{array}{l}\text { Deuxième } \\
\text { version du récit } \\
\text { ( } 21 \text { élèves) }\end{array}$ & $\begin{array}{l}5 \text { cas } \\
(23,80 \%)\end{array}$ & $\begin{array}{l}8 \text { cas } \\
(38,09 \%)\end{array}$ & $\begin{array}{l}13 \text { cas } \\
(61,89 \%)\end{array}$ & $\begin{array}{l}6 \text { cas } \\
(28,57 \%)\end{array}$ & 0 & $\begin{array}{l}2 \text { cas }(9,52 \%) \\
\text { aucune version du } \\
\text { récit écrit }\end{array}$ & $\begin{array}{l}8 \text { cas } \\
(38,08 \%)\end{array}$ \\
\hline
\end{tabular}

Figure 5. - Les formes verbales utilisées dans les trois phases de l'écriture du récit.

Un aperçu rapide de ces données montre que les différences entre la planification et la première version du récit sont minimes. Les enquêtés qui intègrent dans la planification ou dans la première version du récit un temps verbal différent du PS et du PI représentaient entre 33,32\% dans la planification (7 sujets sur 21) et 38,08\% dans les premières versions du récit (8 cas dont 2 avec des usages échoués). Autrement dit, plus de $60 \%$ des élèves n'ont pas fait le transfert prévu des connaissances grammaticales vers l'usage réfléchi, ni dans la planification ni dans la première version du texte.

En analysant les données de la deuxième version du récit, après la tâche de révision entre pairs, nous constatons que les chiffres sont inversés. Cette fois-ci, il y a 13 élèves $(61,89 \%)$ qui ont réussi à incorporer des temps différents de ceux de l'usage spontané, contre 8 cas $(38,09 \%)$ qui n'ont pas réussi à le faire.

Compte tenu de ces résultats, il semble que la révision entre pairs, avec la consigne explicite de revoir l'usage des temps verbaux, consti- 
tue une activité favorable à l'amélioration des récits dans le sens requis. Pour approfondir ces observations, nous nous tournons vers les interactions des élèves au cours de la planification et de la révision de l'histoire.

L'analyse a mis en évidence des différences significatives entre les interactions des élèves pendant la planification et celles de la phase de révision. Pendant les premières, il y a une absence totale de références aux temps du passé. Les élèves se concentrent sur la résolution des questions sémantico-pragmatiques reliées au contenu du texte, comme dans l'exemple ci-dessous (figure 6) :

\begin{tabular}{|c|c|c|}
\hline Tour & ÉLÈVES (MG-SZ-AL3-AL4) & Enseignant (ES) \\
\hline 1 & MG : quel a été le meilleur été de votre vie? & \\
\hline 2 & SZ: je vais mettre le passé & \\
\hline 3 & MG : comment? & \\
\hline 4 & $\begin{array}{l}\text { SZ: Je vais mettre le passé, mais je n'ai encore rien écrit. Des } \\
\text { autres, je ne me souviens pas des autres temps, juste du passé. }\end{array}$ & \\
\hline 5 & $\begin{array}{l}\text { AL3 : Qu'est-ce que vous allez mettre, qu'est-ce que vous } \\
\text { allez mettre? }\end{array}$ & \\
\hline 6 & $\begin{array}{l}\text { MG : Je sûr que celui de Majorque, celui dont je me souviens } \\
\text { le plus }\end{array}$ & \\
\hline \multicolumn{3}{|c|}{$[\ldots] \quad[\ldots] \quad[\ldots]$} \\
\hline 10 & $\begin{array}{l}\text { MG : Regardez, j'ai été la seule chose xxxxx spéciale, c'était } \\
\text { avoir un ticket avec quelqu'un de l'hôtel... c'est ce qui s'est } \\
\text { passé mais je ne mettrai pas ça }\end{array}$ & \\
\hline \multicolumn{3}{|c|}{$[\ldots] \quad[\ldots] \quad[\ldots]$} \\
\hline 14 & $\begin{array}{l}\text { MG : [poursuit avec un ton moqueur] et je vais mettre «J'ai } \\
\text { un ticket avec quelqu'un de l'hôtel» et toi «j'ai acheté une } \\
\text { crème glacée» (rires) }\end{array}$ & \\
\hline 15 & $\begin{array}{l}\text { SZ: Je ne sais pas, j'allais mettre quelque chose, mais je ne } \\
\text { sais pas parce que ça me fait tout drôle... }\end{array}$ & \\
\hline 16 & $\begin{array}{l}\text { MG : xxx sur les plages de Majorque, j'ai seulement mis } \\
\text { les pieds sur le sable la nuit. [Pour le professeur] Je ne sais } \\
\text { pas je n'ai rien fait de spécial, mes vacances sont celles de } \\
\text { Majorque et je ne pense pas qu'on doit mettre aller à la plage } \\
\text { la nuit au lieu de pendant la journée }\end{array}$ & \\
\hline \multicolumn{3}{|c|}{$[\ldots] \quad[\ldots] \quad[\ldots]$} \\
\hline 32 & $\begin{array}{l}\text { MG : attends, ceci est plus Xxxx «rencontrer un gars et partir } \\
\text { pour une promenade romantique le long de la plage, la nuit» }\end{array}$ & \\
\hline \multicolumn{3}{|l|}{$[\ldots]$} \\
\hline 39 & $\begin{array}{l}\text { MG : ça va mieux, n'est-ce pas? C'était une histoire super- } \\
\text { romantique en vérité }\end{array}$ & \\
\hline
\end{tabular}

Figure 6. - Interaction entre élèves. Planification du récit-sélection de l'évènement non habituel et adéquation à la situation discursive. 
Aux premiers tours, les élèves s'intéressent au choix du moment de ce dont ils vont parler. Le tour 10 marque un changement : l'élève MG regarde le fait qui n'était pas habituel, «j'ai été la seule chose xxxx spéciale était avoir un ticket avec quelqu'un de l'hôtel» et ajoute aussitôt, «c'est que c'était ça mais je ne mettrai pas ça». Cette intervention montre l'interrelation des aspects sémantico-pragmatiques de la planification du texte. Pour les élèves, ce qui «peut être dit» (planification pragmatique) est aussi important que le «que dire» (contenu de l'histoire, planification sémantique). À partir du tour 10, les élèves plaisantent sur l'ambiguitté entre «que dire» et «que peut-on dire». Enfin, après plusieurs hésitations, au tour 32, MG a trouvé la solution : «rencontrer un gars et partir pour une promenade romantique le long de la plage, la nuit», une solution qui a reçu l'approbation de ses camarades. Dans cet exemple, il n'y a pas de référence aux temps verbaux. Les élèves parlent du contenu de leur récit et l'activité métalinguistique se concentre sur l'adéquation du sujet à la situation du discours.

Les interactions recueillies pendant la révision, pour leur part, montrent que, majoritairement, les élèves se concentrent sur l'identification des formes verbales des textes qu'ils sont en train de réviser, sur les critères qu'ils utilisent pour le faire (ce qui est verbe ou non...) et sur les dénominations exactes des temps. Dans une deuxième étape, l'identification est complétée par la vérification des temps utilisés. Dans certains cas, l'élève suggère à l'auteur d'insérer dans son texte les temps verbaux manquants. Nous constatons, cependant, que les élèves trouvent qu'il est difficile de proposer des amendements. L'exemple de la figure 7 montre l'interaction de l'enseignant avec l'élève MG, qui propose d'éventuels amendements au texte révisé. MG, après avoir inséré le PS dans le texte ( $«$ J'ai inséré le passé simple, car tous les temps qu'il y avait étaient l'imparfait, c'est-à-dire, je l'ai amélioré avec... mettre un peu », tour 7), se rend compte que l'incorporation du PF implique une réorganisation de la syntaxe («désorganisait un peu la phrase», tour 7 ; «il changeait un peu, et le texte ne semblait que...», tour 9) qu'il n'ose pas réaliser lui-même. Il préfère faire la suggestion à l'auteur du texte («Je peux mettre une observation...?», tour 11). L'interaction avec l'enseignant montre que l'élève est conscient des limites du texte révisé, mais qu'il ne parvient pas à proposer des modifications spécifiques pour mettre en place des formes verbales comme le PF, car cela implique une réorganisation de l'environnement syntaxique de l'énoncé. 


\begin{tabular}{|c|c|c|}
\hline \multirow[t]{2}{*}{ Tour } & Élève (MG) & Enseignant (ES) \\
\hline & $\begin{array}{l}\text { MG : je l'ai amélioré, ça y est. Comme dans son } \\
\text { texte... }\end{array}$ & \\
\hline & & $\begin{array}{l}\text { ES : fais-tu la révision de } \\
\text { AD? }\end{array}$ \\
\hline & MG : oui & \\
\hline & & ES : d'accord, imp- \\
\hline & MG : imparfait... & \\
\hline & & $\begin{array}{l}\text { ES : attends, attends un } \\
\text { moment }\end{array}$ \\
\hline & $\begin{array}{l}\text { MG : passé simple mais... c'est moi qui en ai } \\
\text { mis quelques-uns. Le passé simple c'est moi qui } \\
\text { l'ai mis, tout ce qu'il y avait étaient à l'imparfait, } \\
\text { c'est-à-dire je l'ai amélioré un peu, en mettant } \\
\text { quelque chose, car si on met là... un plus-que- } \\
\text { parfait... ça désorganisait un peu la phrase }\end{array}$ & \\
\hline & & ES : ah, je vois je vois \\
\hline & $\begin{array}{l}\text { MG : ça changeait un peu, et le texte ne semblait } \\
\text { que... }\end{array}$ & \\
\hline & & $\begin{array}{l}\text { ES : de toute façon tu peux } \\
\text { le suggérer ça là bas }\end{array}$ \\
\hline & $\begin{array}{l}\text { MG : oui. Je peux mettre une observation comme } \\
\text { «tu peux, non, il faut mettre...»? }\end{array}$ & \\
\hline & & ES : oui \\
\hline
\end{tabular}

Figure 7. - MG commente avec son enseignant sa révision du récit de $A D$.

La comparaison entre les données relatives aux différentes phases du récit écrit et les interactions lors de la planification et lors de la révision consolide nos observations.

\subsection{Quelles conclusions tirer de l'analyse de la tâche de réflexion?}

Nous mettrons l'accent sur les conclusions générales de l'apprentissage réalisé par les élèves, sur leurs réalisations et leurs difficultés.

Tout d'abord, l'activité intitulée «usage réfléchi» nous permet d'observer l'utilisation par les élèves des formes verbales du passé à partir de deux perspectives : la production (à travers les trois étapes de l'écriture du récit) et l'analyse (la révision entre pairs avant la deuxième version du récit). Comme on l'a dit, l'analyse semble une activité pédagogique intéressante pour provoquer chez les élèves le contrôle délibéré de leurs propres pratiques linguistiques.

La perspective de la production montre que, depuis la planification jusqu'à la deuxième version du récit, les données d'utilisation spontanée 
(PS et PI) et d'usage soutenu (PF et CP) sont inversées. La prise en compte conjointe des deux versions du récit montre que 12 des 21 élèves $(57,14 \%)$ réussissent à utiliser des formes d'usage soutenu (PF ou $\mathrm{CP})$ dans leurs récits, tandis que 7 sur $21(33,33 \%)$ utilisent seulement des formes verbales d'usage spontané (PS et PI). Le PF est la forme du verbe utilisée par tous les élèves capables d'un usage soutenu (12 sur $21,57,14 \%)$. En revanche, seulement la moitié de ces élèves a inséré le $\mathrm{CP}$ dans son récit et on observe une seule tentative pour incorporer, sans succès, le passé antérieur (PA).

Les résultats des élèves quant à leur utilisation des formes de style soutenu (PF, CP et PA) sont cohérents avec les données obtenues dans la conceptualisation. Le PF est la forme verbale la mieux caractérisée et utilisée, suivie par le CP (en tant que futur du passé). Par contre, le PA présente les plus grands obstacles à la caractérisation et l'utilisation.

Du point de vue de l'analyse (révision entre pairs du texte), presque tous les élèves (20 sur 21, 95,23\%) identifient les formes verbales dans les récits de leurs pairs, bien que 12 d'entre eux $(57,14 \%)$ fassent des erreurs portant notamment sur le gérondif ou l'infinitif, considérées comme des formes verbales conjuguées. L'identification des formes verbales est liée à l'évaluation du nombre et du type de formes utilisées dans 16 cas $(76,19 \%)$, mais on ne peut observer des changements ou des suggestions que dans 10 cas $(47,61 \%)$. Les modifications valables s'appliquent à l'intégration du PF ou du CP dans des contextes syntaxiques qui ne nécessitent pas de modifications (remplacement forme à forme). Lorsque les récits sont faibles et exigent des modifications syntaxiques ou discursives, les réviseurs ne proposent que des suggestions d'amendement, comme dans l'exemple de la figure 7 .

Tant au plan de la production qu'au plan de l'analyse, nous constatons l'importance des environnements syntaxico-discursifs, en particulier des expressions temporelles pour indiquer les phases du récit (faits habituels, faits antérieurs ou postérieurs à l'évènement inattendu) pour induire l'usage des formes verbales comme le PI avec la valeur d'habitude, le PF ou le CP.

L'analyse des écrits des élèves dans les différentes phases de la tâche, complétée par l'analyse des interactions en classe et la comparaison avec le modèle du récit, permet d'accéder à l'activité métalinguistique des élèves et fournit des données à la fois sur les résultats et le processus.

Au cours de la tâche d'usage réfléchi, les élèves suivent deux types d'activités métalinguistiques explicites: en tant qu'auteurs de leur propre récit et à titre d'évaluateurs du récit de leurs pairs. Dans le premier cas, 
on observe un contrôle progressif de la production, qui augmente de façon significative après l'examen par les pairs. Dans le second, la situation d'analyse (de «langage opaque») permet à de nombreux élèves de prendre conscience du problème grammatical posé. Cette conscientisation se manifeste principalement dans la deuxième version du récit.

$\mathrm{Au}$ cours de la phase de planification, les aspects formels ne sont pas contrôlés par les élèves - à une exception près - , qui se consacrent d'abord à résoudre la configuration globale du texte dans ses niveaux sémantiques et pragmatiques, et qui dans ce cas se concentrent sur leur activité métalinguistique, liée à l'usage spontané de la langue. Cela explique la prédominance des formes verbales propres aux usages oraux des élèves.

En ce qui concerne l'écriture du texte (première ou deuxième version), réalisée à la maison, l'activité métalinguistique des élèves répond essentiellement à quatre situations : l'augmentation, le maintien ou la disparition de formes verbales d'usage soutenu et la persistance des formes d'usage spontané. L'augmentation et le maintien de formes verbales d'usage soutenu mettent en évidence les acquis des élèves, tandis que la disparition des formes verbales d'usage soutenu ou la persistance de l'usage spontané nous permettent de cibler les obstacles qui se présentent à l'élève au cours de la tâche.

La révision entre pairs, quant à elle, implique un changement dans la position de l'énonciateur par rapport à celle de lecteur qui révise, favorisant ainsi la prise de distance nécessaire pour observer la forme de la langue, en l'occurrence, l'usage des formes verbales du passé.

Les remarques des élèves lors de la révision relèvent de trois types d'activités : l'identification des formes verbales du texte, l'évaluation des formes les plus utilisées ou du bon usage et la modification. L'identification n'est pas strictement une révision, mais c'est une première étape nécessaire, car elle concentre l'attention sur les objectifs de la tâche et contribue à créer la situation de «langage opaque » qui favorise la réflexion métalinguistique postérieure. L'évaluation implique la détection du problème analysé et son premier diagnostic. La modification implique toutes les sous-opérations du processus de révision (la détection et l'identification du problème, la comparaison avec un modèle préexistant, ce qui conduit à un diagnostic, et la prise de décisions dans un contexte syntaxico-sémantique déterminé précédemment).

Les interactions des élèves avec le professeur nous permettent de mieux connaitre leurs réflexions pendant le processus, qui ne se reflètent pas toujours dans les versions finales. Ces pensées verbalisées fournissent des informations utiles sur les acquis des élèves et sur leurs 
difficultés au cours de la tâche, d'où l'importance de leur analyse pour compléter les données fournies par les annotations écrites et pour accéder à l'activité métalinguistique des élèves. La révision permet aux élèves de voir les aspects qui seraient autrement passés inaperçus dans leur utilisation de la langue, comme l'utilisation (ou le manque d'utilisation) d'une forme verbale donnée. En ce sens, l'identification, l'évaluation et la modification impliquent explicitement une réflexion métalinguistique que les élèves ne peuvent pas toujours verbaliser «correctement». La révision crée chez les élèves la conscience métalinguistique par rapport à l'objectif grammatical posé et cette prise de conscience se manifeste dans le contrôle de l'usage quand ils réécrivent leurs propres récits.

La révision entre pairs de l'usage des formes verbales du passé présente également des obstacles. La modification des formes verbales d'un texte doit faire face à l'obstacle du manque de maitrise des formes verbales d'usage soutenu par les réviseurs, dans des cas tels que le PF ou le $\mathrm{CP}$, ce qui explique les remplacements erronés ou non pertinents. Les élèves trouvent qu'il est difficile de réviser ce qui ne fait pas partie de leur forme normale d'expression. C'est sans doute pour cette raison qu'ils demandent l'accord du professeur et qu'ils mettent l'accent sur l'identification des formes verbales. Dans cette situation, l'interaction entre pairs a ses limites. Les substitutions erronées ou non pertinentes et les cas où on identifie et on évalue seulement, montrent que la plupart des élèves révisent les textes avec les critères de leur propre utilisation spontanée. À ce moment-là, ils ne font pas le transfert de la réflexion grammaticale explicite de la tâche de conceptualisation.

\section{Quelques considérations finales sur l'enseignement grammatical comme usage réfléchi de la langue}

Comment résumer l'analyse de ces données? Nos résultats montrent tout d'abord que le développement des connaissances grammaticales doit tenir compte : a) de la nécessité d'échelonner la complexité des concepts ; b) des liens entre les concepts et les contextes énonciatifs ; c) de l'importance de la maitrise fonctionnelle; d) de la relation entre le langage courant et le métalangage. Nos résultats montrent également que le transfert des savoirs déclaratifs à l'usage réfléchi de la langue exige des médiations didactiques en classe, parmi lesquelles il faut souligner l'interaction des pairs et la révision avec des objectifs fixés. 
La relation entre la conceptualisation des formes verbales du passé et leur usage réfléchi n'est pas directe et ne se produit pas spontanément. Pour induire didactiquement le transfert d'un savoir théorique à un savoir-faire réfléchi, il faut des situations didactiques dans lesquelles le professeur partage le contrôle avec les élèves, telles que l'écriture dans la classe ou la lecture et l'évaluation des textes écrits par les élèves, selon des critères établis. Ce type de situations didactiques permet l'évaluation formative, qui favorise une démarche progressive du contrôle externe à la maitrise des usages complexes du langage.

L'enseignement des formes verbales isolées, envisagées comme éléments d'un paradigme, ne permet pas aux élèves d'améliorer leur maitrise de l'usage, qui reste réduite dans la plupart des cas aux formes d'utilisation spontanée (orales). L'enseignement des formes verbales du passé, notamment les formes verbales relatives, ne peut se réaliser sans tenir compte des contraintes inhérentes aux contextes syntaxico-discursifs concernant les notions d'antériorité et de postériorité par rapport à l'action principale. Les expressions temporelles jouent un rôle à cet égard, compte tenu des valeurs aspectuelles et temporelles impliquées.

L'enseignement de la grammaire liée à l'utilisation réflexive du langage requiert des approches méthodologiques qui combinent l'analyse et le contrôle, lire et écrire, et qui permettent aux élèves de verbaliser leur activité métalinguistique dans les interactions avec leurs pairs et le professeur. Cette verbalisation facilite le processus d'internalisation qui conduit de l'interpsychologique (ce qu'on peut effectuer à l'aide d'autres) à l'intraspsychologique (ce que chacun est capable d'effectuer par lui-même, indépendamment), par rapport à la réflexion sur l'usage des langues.

Le cadre des séquences didactiques de grammaire (SDG) intègre les exigences méthodologiques précédentes et permet des approches dans lesquelles l'élève doit s'approprier un savoir grammatical déclaratif et un usage réfléchi de la langue. Par conséquent, cette démarche permet à nos yeux de donner un sens différent au travail grammatical effectué en classe. 


\section{RÉFÉRENCES BIBLIOGRAPHIQUES}

BARTH, B. M. (2004). L'apprentissage de l'abstraction (nouvelle édition revue et augmentée avec une préface et une postface inédites). Paris : Retz.

Benveniste, É. (1966). Les relations de temps dans le verbe français. Dans É. Benveniste, Problèmes de linguistique générale 1 (p. 237-250). Paris : Gallimard.

CAmps, A. (1996). Proyectos de lengua entre la teoría y la práctica. Cultura y Educación, 2, 43-57.

CAmps, A. (2000). Aprendre gramàtica. Dans A. Camps \& M. Ferrer (dir.), Gramàtica a l'aula (p. 101-118). Barcelone : Graó.

Camps, A., Milian, M., Pérez, F., Ribas, T \& Castelló, M. (2001). Los conceptos gramaticales de los alumnos de secundaria: el pronombre personal. Dans A. Camps (dir.), El aula como espacio de investigación y reflexión (p. 161-180). Barcelone : Graó.

Camps, A. (coord.), Guasch, O., Milian, M. \& Ribas, T. (2005). Bases per a l'ensenyament de la gramàtica. Barcelone : Graó.

CAmps, A. \& ZAYAS, F. (dir.). (2006). Secuencias didácticas para aprender gramática. Barcelone : Graó.

FisHeR, C. (2004). La place des représentations des apprenants en didactique de la grammaire. Dans C. Vargas (dir.), Langue et études de la langue (p. 383-393). Aix-en-Provence : Publications de l'Université de Provence.

Flick, U. (2007). Introducción a la investigación cualitativa. Madrid: Morata-Fundación Paideia Galiza.

Kilcher-Hagedorn, H., Othenin-Girard, C. \& de Weck, G. (1987). Le savoir grammatical des élèves. Berne : Peter Lang.

Lemke, J. L. (1997). Aprender a hablar ciencia. Barcelone : Paidós.

Nunan, D. (2002). El diseño de tareas para la clase comunicativa. Madrid : CUP.

PigGot, T. D. \& BARR, R. (2002). Designing programmatic interventions. Dans M. L. Kamil et coll. (dir.), Methods of literacy research (Vol. III, p. 99-108). New Jersey/Londres : Lawrence Erlbaum.

Rodríguez Gonzalo, C. (2008). La organización del aprendizaje lingüístico y literario. Dans C. Rodríguez Gonzalo (dir.), La lengua escrita y los proyectos de trabajo (p. 19-42). Valence : Perifèric.

Rodríguez Gonzalo, C. (2011a). El saber gramatical sobre los tiempos del pasado en alumnos de $4^{\circ}$ de Secundaria Obligatoria (Thèse de doctorat, Université de Valence, Espagne). Repéré à http://tesisenred.net/ handle/10803/81311 
RodríGuez GonZAlo, C. (2011b). Las relaciones entre conceptualización y uso reflexivo en la enseñanza de la gramática. Una investigación empírica sobre los tiempos del pasado. Dans M. ${ }^{a}$ P. Núñez Delgado \& J. Rienda (dir.), La investigación en Didáctica de la Lengua y la Literatura: Situación actual y perspectivas de futuro (p. 435-455). Madrid : SEDLL.

Stake, R. E. (2005). Qualitative Case Studies. Dans N. K. Denzin \& Y. S. Lincoln (dir.), The Sage handbook of Qualitative Research (p. 443466). Thousand Oaks : Sage.

WeInRICH, H. (1974). Estructura y función de los tiempos en el lenguaje (traduit par F. Latorre). Madrid : Gredos.

YIN, R. K. (2003). Case study research. Design and methods. Newbury Park, CA : Sage. 